\title{
Research on Traditional Chinese Freehand Paper-cut
}

\author{
Jinsong $\mathrm{Yu}$ \\ School of Art and Design, Wuhan University of Science and \\ Technology, \\ Wuhan, China \\ Email: yujinsong@wust.edu.cn
}

\author{
Shengli Chen \\ School of Design, Jianghan University, \\ Wuhan, China \\ Email: 527395960@qq.com
}

\begin{abstract}
Things are me, and I am the things" is the universe view theory that human is an integral part of nature or the identity of objects and self. It determines that Confucianism and Taoism have become the basis of philosophy and aesthetics in Chinese traditional art, and form a unique freehand artistic style. Paper-cut is not only an ancient Chinese traditional folk art but also one of the most popular folk art in China. As an art form, Chinese folk paper-cut is the cultural carrier of Chinese primitive philosophy, which is manifested in all aspects of folk culture. When the freehand artistic style is applied in the process of creating paper-cut artwork, it demonstrates the ultra-space-time art forms in the three aspects: conceptual composition, conceptual shapes, and conceptual colors, and arbitrary decoration is used to emphasize endless vitality of life. This art is a veritable freehand paper-cut with arbitrary expression, rich cultural connotation and strong visual impact.
\end{abstract}

\section{Keywords-Freehand; Paper-cut; Concept; Vector}

\section{INTRODUCTION}

\section{A. Freehand}

The freehand brushwork in traditional Chinese painting (characterized by vivid expression and bold outline), refers to the technique or method of Chinese painting and belongs to the simple category of painting [1]. Asked to focus on depicting the state of mind and charm of the image by using the concise pen and ink, hence the name. Freehand brushwork "is also a very special concept of Chinese aesthetics, which represents an aesthetic concept and artistic spirit. It focuses on portraying and depicting the inner min and spiritual demeanor of objects, opposing mechanical imitation and objective reproduction of objects, and trying to transmit the subjective psychological factors of spirit and emotion, aesthetic perception into the object of "humanity." It can be said that the term "freehand brushwork" originated from the characteristics of Chinese culture, and is a cultural identity and national consensus of Chinese people. It is a universal and lack of the aimed characteristic concept to Chinese culture, all Chinese cultural and art forms can be labeled as "freehand brushwork"; "Freehand" is also the soul and highest level of Chinese culture and art.

\section{B. Paper-cut}

Paper-cut is an ancient Chinese traditional folk art, but also one of the most popular folk art in China. According to the paper-cut history of archeology, paper-cutting can be traced back to the sixth century $\mathrm{AD}$, but people think it's actually started a few hundred years earlier than this. The main material for paper cutting is paper. Before paper appearing, there was no real paper-cut. But there are the cutting and engraving of other sheet materials before paper invented. Historical relics and records show that during 1950-1952, an arc-shaped decorative ornament engraved with silver foil was found at the excavated site of the Warring States Period at Guwei Village, Huixian County, Henan Province, and some gold sheet decoration was unearthed in Huangdian, Hunan. These hollow ornaments made of silver foil, although it can't be said as paper cutting, in the engraving technology and artistic style, it can be said that has formed the predecessor of paper cut art. In the vicinity of Huoyan Mountain in Turpan, Xinjiang Uygur Autonomous Region has unearthed five groups of paper-cut circle flowers. This is a beautiful pattern cut by paper, and it is also the earliest paper-cut objects found in our country and well documented. According to research, the Chinese paper-cut originated in the north and spread to the south, the southern paper-cut was evolved. View from the point of style, the traces of ancient totem worship can be found from northern paper-cut through its bold, rugged style, most of South paper-cut are closely linked to daily life, so that looks fine and graceful. Paper cutting was originally used for religious ceremonies. In the past, people often used paper to make various forms of object images and portraits to bury with the dead or burn at funerals. This custom is still seen sometimes outside China. Generally, the symbolic nature of Paper-cut art is a part of the ritual; in addition, paper-cuttings are also used as ornaments for the sacrificial offerings of ancestors and gods. Now, paper-cut is more used for decoration. Paper-cuts can be used to embellish walls, windows, doors, houses, mirrors, lights, and lanterns, but also to embellish gifts, and even paper-cuts can be presented to others as gifts. People used to make paper-cut to be the model for embroidery and spray-painted art. However, some people think that paper-cut originated from ancient practical art.

In short, paper-cut art is one of the most representative forms of art in China's folk culture and the arts, with the widest masses of people, the most distinctive regions, the most 
abundant historical and cultural connotations. Its cultural connotation and artistic form are the historical and cultural deposits of the Chinese nation from the primitive society up to today for six-seven thousands years. Therefore, its cultural value far beyond the art of paper-cut itself [2].

\section{TRADITIONAL CHINESE PAPER-CUT}

\section{A. Unique modeling system}

Chinese traditional paper-cutting has its own unique modeling system, not only because of its strong artistic characteristics of decorative deformation but also because of this decorative form of artistry, which is an art form determined by China's original philosophy system, artistic system and aesthetic concept [3]. This art form reflects the concept of the creative group, and it is these concepts that give works of paper-cut art unique connotations created by the people. These ideas can be divided into the following categories.

(1)The view of modeling in Yin and Yang. Yin and Yang (Taoist Philosophy), the two opposing principles in nature, the former feminine and negative and the latter masculine and positive. The concept of yin and yang originated from the view of nature of the ancient Chinese people. The ancients observed all kinds of natural phenomena in the world, such as heaven and earth, sun and moon, day and night, cold and heat, men and women, and so on, summarized in a philosophical way of thinking the concept of "yin and yang". The Paper-cuts for Window Decoration of "Sky Opened by Rat Biting" (fig. 1) is the common paper-cut theme in Shanxi folk. The rat is a child of God, and it represents the same connotation of fish, both belong to the multiplication symbol. The meaning of this Paper-cut for Window Decoration expression is that the buckled bowl represents the universe, combination of heaven and earth; the rats who is the son of God lies in it; everything includes human beings are formed after the rats biting to open the sky and drilling out. The buckled bowl of paper-cut demonstrates the modeling language of art in the intersection of heaven and earth, Yin and Yang, men and women.

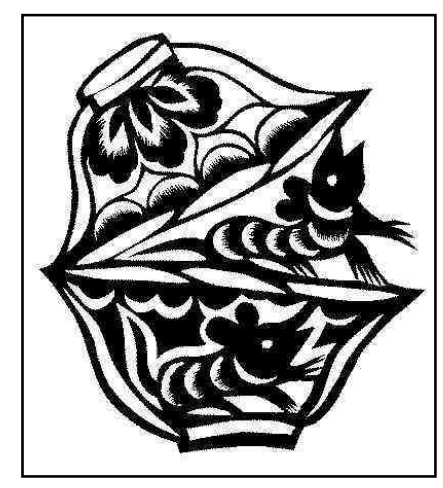

Fig. 1 The Paper Cut of "Sky Opened by Rat Biting".

(2)The view of modeling in five elements. The Five Elements [4] is a systematic conception of Taoism in ancient China. Ancient Chinese philosophers used the Five Elements Theory to illustrate the formation and interrelationships of all things in the world. It emphasizes the whole, designed to describe the movement of things and transformation. Five Elements is the original system theory. Chinese folk artists do not follow the perspective principle of traditional Western, nor do they follow the perspective principle of peculiar scatterpoint in Chinese painting, but instead of modeling with the concept of Chinese origin philosophy [5]. In the Paper-cuts for Window Decoration of "Rats Steal Oil"(fig. 2), the bottle means the matrix and the mouse is the symbol of reproduction. In the paper cutting, the mouth of the oil bottle is an upward arc to represent the heaven, while the bottom of the bottle is a horizontal and straight line to imply the ground. The whole bottle means a universal matrix of the round heaven and the square ground. This artistic method of anti-perspective is not the geometric composition of round and square from the western modern art, but the form composition of the concept of Chinese philosophy.

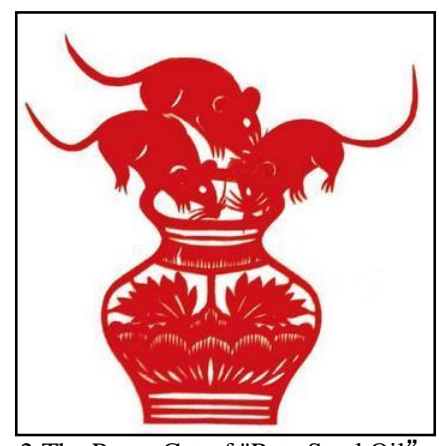

Fig. 2 The Paper Cut of "Rats Steal Oil"

(3)The view of modeling in Totems. Totem refers to the worship of a particular animal or plant and other objects as a kinship or other special relationship with the clan. It is the original form of the primitive religion. The primitive people believed that a relatives or other special relations exist between a clan and some kind of animal or other natural objects, generally animals. The tigers shown by the creators of Chinese folk art are not tigers of a natural form in specific temporal and spatial but tigers of a super-temporal concept and a totem tiger protecting the clan community. There are two kinds of tigers such as "The Aihu"(fig. 3) with chubby legs to protect dolls to play; "The Downhill Tiger"(fig. 4) with a fierce look, four legs kicking before and after to protect houses. Both of these two folk tiger patterns are not the tigers of natural form in a specific moment of time and space.

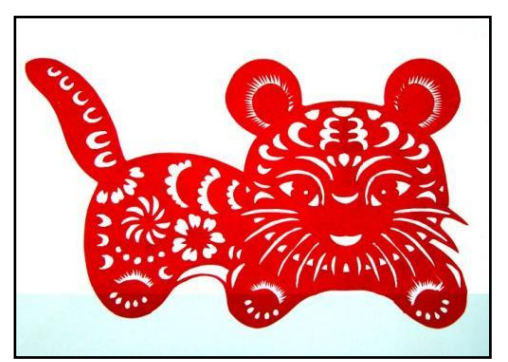

Fig. 3 The Paper Cut of "The Aihu". 


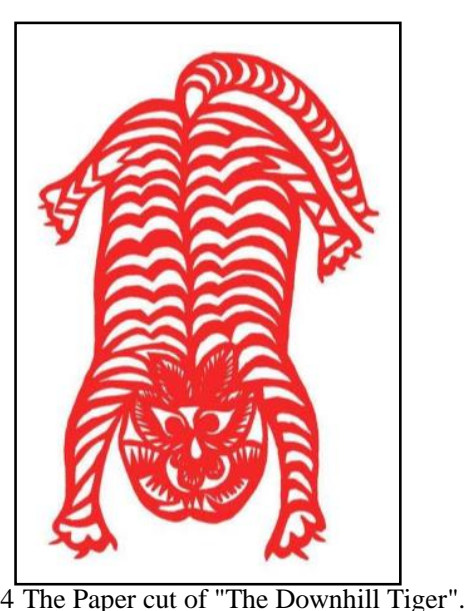

(4)The view of modeling in hyperspace. The content described by hyperspace is not based on the realistic sensory world but based on the application and perspective of the mysterious wisdom of human beings to expresses, creates, reconstruct space-time to display the meta-wisdom. The symbols of living plants, animals and the gods of all things are used to replace the no life decoration such as lines, black and white, light and shade changes. For example, in some papercut artworks, it can be seen that the pig head with the concept of god replace the pearls in "Two Dragon Play Pearls"; peonies decorated the roof instead of inanimate tiles; Lotus decorated fish instead of inanimate fish scales; flowers and animal patterns Decorated human's clothing and animal fur, etc., to highlight a song of cosmic and life. This is determined by the emotional temperament and psychological qualities of Chinese folk art creators, which can't be achieved by the artistic creation of a particular perspective and time [6].

It can be seen that the paper-cut is full of the philosophical meaning of Yin and Yang concept, concept of Five Elements, Totem concept, Super Space-time view, helps people to understand folk paper-cut art and deepen their understanding of the characteristics of the art form.

\section{B. Personalized color system}

In addition to its own unique modeling system of the traditional Chinese paper-cut, there is a very personal color system. Simultaneously, this color system embodies people's understanding of the world and the universe, reflects people's thinking and conception [7]. It includes the following aspects.

(1)The view of color in life-and-nature. Life-and-nature refers to a concept that all things and creatures are endless, cyclic. Red is the color worshiped by the Chinese nation. It spreads to this day from 40,000 years ago, the caveman sprinkles red mineral around the dead to pray for immortal soul and eternal life. Red color, as a symbol of the life worship, it stems from the worship of the sun, worship of fire, Understanding of blood that represent life. The traditional Chinese New Year is a festival of red oceans. Many people wear red clothes, red hat and even wearing all red; there is the red words, the red poetic couplet, red lanterns everywhere. Red is also the color of avoiding evil in the eyes of the Chinese people. All demons and ghosts who threaten the existence of life are afraid of red color. Therefore, cinnabar and dark red paper have become the colors of choice for Chinese people in town.

(2)The view of color in yin-and-yang. Red color represents life multiply of Male and female, white color shows the death of a person. It originates from the red sun of warm and the white snow of the withered winter. In wedding, the male dress in red and the female dress in green imply the combination between the sun's red and grass green crop. According to this view of Yin and Yang, all ethnic groups have their own different color combinations, such as the ethnic of tiger totem and worship, the black tiger is regarded as the god, the father; the white tiger as the ground, the mother; black and white represents the color combination of heaven and earth, Yin and Yang.

(3)The view of color in Five Elements and Eight Diagrams. The view of Five Elements in color of the Chinese nation represents Chinese people's concept of space and time. The concept of "Five Elements" in East, West, South, North, and center is the concept of space for Chinese. Its color combination is the blue in oriental, white in western, red in southern, black in northern and yellow in the center, Running in the four seasons of spring, summer, autumn, and winter, it is the concept of time for Chinese people. It matches the five elements and five colors, that is the color combination of blue in spring, red in summer, yellow in long summer, white in autumn, black in winter; it matches the five lines of the totem God, that is the oriental dragon and the blue; the western white tiger and the white; the southern Suzaku and the red, the Northern basalt and the black; central yellow.

The above shows that the color of paper-cut art has become a symbol of the connotation of a substitute. This morality also reflects people's simple, sincere and frank psychology.

\section{TRAditional Chinese PAPER-CUT : A FreEhand PAPER-CUT}

Paper-cut, as a form of Chinese folk art, is the cultural carrier of the Chinese origin philosophy, which is manifested in all aspects of folk culture. The working women group is the main creative group in this art category. They apply the original philosophical view, philosophical symbols and passwords to express their understanding of the universe. "Different nation and Different region have different symbols passwords, and their creators of different temperament and cultural accomplishment, use different artistic languages to express different art forms" [8].

According to the western ancient Greek philosophical point of view, "art is the imitation of nature" and "the artist should vividly imitate things" [9], then the task of the artist is to imitate the object world naturally, and the most vivid imitation is to achieve the highest Realm, so artists must study the structural laws and color laws of natural objects as well as their texture, volume, sense of space, and sense of volume in order to achieve the highest imitation of the natural object. The imitation theory of art creation by the dichotomy between subject and object has been the philosophical and aesthetic foundation of western art for two thousand years. 
The Chinese philosophical system and the art system are the combinations of man and nature, the unity of subject and object. Chinese artists use the natural object to express their personal feelings in their artworks. From the perspective of Chinese artist, they believe that the thing is me, and I am the thing. It is the philosophical and aesthetic foundation of traditional Chinese fine arts and its freehand artistry, and this kind of expression of Confucianism and Taoism is determined by the harmony between man and nature, the unity of object and subject. Chinese painting, whether it is freehand brushwork or fine brushwork, is completely different from the imitation theory of Western art creation. Therefore, although there is no such thing as "freehand paper-cut" in Chinese folk papercutting, judging from the philosophical and aesthetic foundation of Chinese traditional art, it is reasonable to refer to traditional Chinese paper-cut as "the freehand paper-cut".

\section{CONCLUSION}

As an artistic method, freehand is a typical property of traditional Chinese painting, which characterized by vivid expression and bold outline. Furthermore, it is a specific concept of Chinese aesthetics that reflects Chinese artists' unique view of modeling and thought level. It represents a kind of aesthetic concept and artistic spirit, emphasizes to convey the spirit, and gives the object a "personification" by moving the subject's aesthetic perception into the object's aesthetic object [10]. The word "freehand" originates from the characteristics of Chinese culture and is the cultural consensus of the Chinese nation. It is a concept of universal significance for Chinese culture, all Chinese culture and art forms can be dubbed as "freehand" that is also the soul and highest level of Chinese culture and art.

Chinese folk paper-cutting is one of art form of Chinese culture, it embodies a complete system of philosophy and art, which is based on China's source philosophy system. This is a complete, prehistoric philosophical system that contains the simplest conceptions: the combination of Yin and Yang, the conversion of all things, the endless growth of all things, which makes people in the process of creating paper-cut artwork, whose art forms are the conceptual composition, conceptual shapes, and conceptual colors over time and space, and use arbitrary decoration to emphasize endless vitality of life.

Based on the artistic method of freehand, paper-cut art with arbitrary expression, rich cultural connotation, the strong visual impact can be named as a veritable freehand paper-cut, it provides a new perspective for people to understand traditional Chinese art forms from the angle of Chinese ancient philosophy.

\section{ACKNOWLEDGMENT}

The authors would like to thank Professor Yong Zeng for providing resources for research opportunity, research equipment, and research methodology.

\section{REFERENCES}

[1] Peiyi Chen, the Boundary of Freehand, Sculpture Magazine .p34-36. Total No. 64, 2008. (In Chinese)

[2] Jinjuan Zhang, Paper-cut, China traveling press, 2015. (In Chinese)

[3] Qian Wang, Qun Liu, The application of Chinese Paper-Cut Modeling in Graphic Design, Art Education Research, p84. Total No. 5, 2017. (In Chinese)

[4] Taoist Philosophy, Retrieved July 2017, from https://baike.baidu.com/item. (In Chinese)

[5] Gaodi Tai, China paper cut - the brilliant modeling art in paper, World Heritage.p122-127. Total No. 1, 2017. (In Chinese)

[6] Shanxi Intangible Cultural Heritage: Zhongyang Paper Cutting, $\begin{array}{lcl}\text { Retrieved July } & 2016, & \text { from }\end{array}$ https://zhidao.baidu.com/question/2011441867036459188.html.(In Chinese)

[7] Johannes Eaton, Color Art, Du Dingyu Translation, Shanghai People's Fine Arts Publishing House, 1985.

[8] Zhilin Jin, Chinese Folk Art, China Intercontinental Press, 2004. (In Chinese)

[9] Chenyuan Guo, Resesrch on modeling character of Shanxi Paper-cut, Home of Dreamer, p139. Total No. 24, 2015. (In Chinese)

[10] Xiaotao Liu et al., Freehand Sculpture Frontier, Sculptures Magazine .p34-36. Total No. 64, 2008. (In Chinese) 